\title{
Rechtsprechung
}

\section{Keine Sicherstellung einer Buddha-Statue nach dem KGSG bei Unsicher- heit über Ausfuhrzeitpunkt und Rechtslage im Herkunftsstaat}

VG Karlsruhe, Urteil vom 24.6.2020, 5 K 7747/18

\section{Tenor}

1. Der undatierte Bescheid des Ministeriums für Wissenschaft, Forschung und Kunst Baden-Württemberg (Datum des in den Behördenakten enthaltenen Entwurfs: 12.06.2018), mit welcher die Sicherstellung der am 30.05.2018 vom Zollamt Heidelberg angehaltenen Buddha-Statue verfügt wurde, wird aufgehoben.

2. Die Beklagte wird verpflichtet, diese Buddha-Statue (beschrieben unter Nummer 267 des Auktionskatalogs des XXX, USA für die Versteigerung am 7. April 2018, $10 \mathrm{Uhr}$ und wie in Nr. 2 des Klageantrags der Klageschrift abgebildet) an den Kläger herauszugeben.

3. Der Beklagte trägt die Kosten des Verfahrens.

\section{Tatbestand}

Der Kläger wendet sich gegen die Sicherstellung einer Buddha-Statue.

Am 30.05.2018 wurde das Ministerium für Wissenschaft, Forschung und Kunst Baden-Württemberg (im Folgenden: Ministerium) vom Zollamt Heidelberg über die Anhaltung einer Buddha-Statue unterrichtet. Diese hatte der Kläger über das in den USA befindliche Auktionshaus XXX für 1.651,00 US-\$ ersteigert. Die Statue wurde in der Rechnung vom 07.04.2018 als "Gilt Lacquered Wood Figure of Buddha“" (Lot \# 0267) spezifiziert. In seiner mit E-Mail vom 30.05.2018 übersandten Auskunft teilte das Badische Landesmuseum mit, dass die Statue echt zu sein scheine, eine Datierung in das 17./18. Jahrhundert nach Chr. sei nicht auszuschließen, als Herkunftsländer kämen Tibet bzw. das restliche China oder Myanmar in Betracht.

Mit an den Kläger adressierter undatierter Verfügung, die sich als auf den 12.06.2018 datierter Entwurf in den Behördenakten befindet, ordnete das Ministerium gem. § 33 Abs. 2 KGSG die Sicherstellung der Buddha-Statue mit folgender Begründung an: Es handele sich nach aktuellem Kenntnisstand um eine echte Buddha-Statue aus dem 17./18. Jahrhundert nach Chr. Wahrscheinliche Herkunftsländer seien China oder Myanmar. Eine Ausfuhrgenehmigung oder sonstige Bestätigung des Herkunftsstaates, dass die Statue rechtmäßig von dort habe ausgeführt werden können, liege nicht vor. Nach § 33 Abs. 1 Nr. 1b KGSG sei Kulturgut sicherzustellen, wenn der hinreichende Verdacht bestehe, dass es entgegen einem Ver- bot nach § 28 KGSG eingeführt werden solle. § $28 \mathrm{Nr}$. 1 KGSG verbiete die Einfuhr von Kulturgut, wenn es von einem Vertragsstaat, für den das UNESCO-Übereinkommen über Maßnahmen zum Verbot und zur Verhütung der rechtswidrigen Einfuhr, Ausfuhr und Übereignung von Kulturgut bindend sei, als nationales Kulturgut eingestuft oder definiert worden sei und unter Verstoß gegen dessen Rechtsvorschriften zum Schutz nationalen Kulturguts aus dessen Hoheitsgebiet verbracht worden sei. Vorliegend bestehe bei beiden in Frage kommenden Herkunftsländern ein solcher Verdacht. Die Volksrepublik China sei seit 28.11.1989 UNESCO-Vertragsstaat. Bei der Statue handele es um chinesisches Kulturgut gem. Art. 2 Abs. 3 Kulturgutschutzgesetz der Volksrepublik China vom 19.11.1982, zuletzt geändert am 24.04.2015. Für Kulturgut, das vor 1911 geschaffen worden sei, bestehe nach nationalen chinesischen Rechtsvorschriften ein absolutes Ausfuhrverbot. Myanmar sei seit 05.09.2013 UNESCO-Vertragsstaat. Die Statue stelle nach Sec. 2 (a) i.V.m. Sec. 4 The Protection and Preservation of Antique Objects Law (2015) ein antikes Objekt dar, dessen Ausfuhr gemäß Sec. 14 dieses Gesetzes verboten sei. Die Statue sei auch gem. § 33 Abs. 1 Nr. 1 (sic.) KGSG sicherzustellen, weil bei der Einfuhr die nach $\S 30$ KGSG erforderlichen Unterlagen nicht vorgelegt worden seien. Vermerke, wann die Verfügung abgesandt wurde, finden sich nicht in der Akte.

Mit am 16.07.2018 beim Verwaltungsgericht Stuttgart eingegangenem Schriftsatz hat der Kläger Klage erhoben; dieses hat das Verfahren mit Beschluss vom 01.08.2018 - 8 K 7608/18 - an das Verwaltungsgericht Karlsruhe verwiesen.

Zur Begründung trägt der Kläger vor: Er habe die BuddhaStatue über das Internet am 08.04.2018 bei einem in Kalifornien, USA ansässigen Auktionshaus für einen Zuschlagspreis von 1.300 US-\$ erworben. Bei dem Auktionshaus handele es sich um einen kleineren Betrieb, dessen Angebote in der Regel aus Nachlassverwertungen stammen dürften. Er habe die Buddha-Statue als „Dekorationsobjekt" für sich selbst erworben. Der Auktionskatalog habe keine Hinweise zu Alter oder Herkunft des Stückes enthalten. Auskünfte habe das Auktionshaus - auch im Nachhinein - nicht erteilt. Es werde bestritten, dass die Statue aus China oder Myanmar und aus dem 17./18. Jahrhundert stamme. Unterstelle man, dass die Statue tatsächlich alt sei, könnte es sich genauso gut um eine japanische Arbeit handeln. Japan verbiete die Ausfuhr von Kulturgut nur, wenn es in einem förmlichen Verfahren durch die zuständige Behörde zu einem Register nationalen Kulturguts hinzugefügt worden sei. Dies sei bei der steitgegenständlichen 
Statue nicht der Fall. Es gebe zudem keinerlei Anhaltspunkte dafür, dass die Statue unrechtmäßig aus dem Ursprungsland ausgeführt worden sei. Die Tatsache, dass sie Gegenstand einer Nachlassauktion gewesen sei und es insbesondere im Auktionskatalog keinerlei Hinweise auf ihre Herkunft gegeben habe, spreche dafür, dass sie sich schon länger in den USA befunden habe. Die Statue müsse, wenn sie aus China, Myanmar oder Japan stammen sollte, schon vor geraumer Zeit, jedenfalls aber vor dem 26.04.2007 in die USA gelangt sein. Der Beklagte trage die Beweislast dafür, dass die Statue erst nach diesem Zeitpunkt ihr mögliches Ursprungsland verlassen habe. Das Ministerium habe zu einem unbekannten Zeitpunkt die angegriffene Verfügung erlassen, welche ihn am 16.06.2018 auf dem Postweg erreicht habe.

Die vom Beklagten herangezogene Ermächtigungsgrundlage des $\S 33$ Abs. 1 Nr. 1 b i.V.m. § 28 Nr. 1 KGSG sei verfassungswidrig. Sie verstoße gegen Art. 14 Abs. 1, Art. 20 Abs. 3, Art. 19 Abs. 4, Art. 103 Abs. 2 und Art. 2 Abs. 1 GG. Tatbestandsvoraussetzung des $\S 28 \mathrm{Nr}$. 1 KGSG sei, dass das Kulturgut von einem Mitgliedstaat oder Vertragsstaat als nationales Kulturgut eingestuft oder definiert und unter Verstoß gegen dessen Rechtsvorschriften zum Schutz nationalen Kulturgutes aus dessen Hoheitsgebiet verbracht worden sei. Damit würden ausländische Normen mittelbar Tatbestandsvoraussetzung. Da § 28 Nr. 1 über § 33 Abs. 1 Nr. 1 b KGSG massiv in Eigentumsrechte eingreife, müsse eine vollumfängliche richterliche Überprüfung der Tatbestandsvoraussetzungen möglich sein.

Der Beklagte berufe sich im Hinblick auf die vermeintliche Herkunft der Buddha-Statue aus China auf chinesische Rechtsvorschriften, die für Kulturgut, welches vor 1911 geschaffen worden sei, ein absolutes Ausfuhrverbot begründeten. Der zitierte Art. 2 Abs. 3 des Kulturgutschutzgesetzes der Volksrepublik China vom 19.11.1982 sehe dies aber gerade nicht vor. Auf der Internetseite der Beauftragten der Bundesregierung für Kultur und Medien unter www.kulturgutschutz-deutschland.de werde im Länderbericht zu China zudem darauf hingewiesen, dass „die aktuellen Gesetzesfassungen nicht in Gänze frei einsehbar sind". Den dort gemachten Auskünften lägen daher „Rechtsauskünfte von Staatsseite" zugrunde. Es bleibe daher unklar und auch nicht nachprüfbar, aufgrund welcher chinesischen Vorschrift der Beklagte von einem Ausfuhrverbot für alle vor 1911 geschaffenen Gegenstände ausgehe, wobei unterstellt werde, dass die Statue tatsachlich aus China und aus dem 17./18. Jahrhundert stamme. Es wäre mit grundlegenden rechtsstaatlichen Grundsätzen nicht vereinbar, wenn der Rechtsunterworfene die Sicherstellung seines Eigentums aufgrund einer Norm hinnehmen müsste, die ihm weder konkret genannt werde, noch anderweitig zugänglich sei. Zudem bestünden auch weitere Bedenken gegen die VerfassungsmäBigkeit der Ermächtigungsgrundlage, weil § 28 Nr. 1 i.V.m. § 83 Abs. 1 Nr. 2, 3 KGSG strafrechtlich bewehrt sei und die aufgezeigte Unklarheit hinsichtlich des Tatbestandes auch einen Verstoß gegen Art. 103 Abs. 2 GG darstelle.
Selbst wenn die Ermächtigungsgrundlage verfassungsmäßig wäre, lägen hier die Tatbestandsvoraussetzungen von $\S 33$ KGSG nicht vor. Es bestehe kein hinreichender Verdacht dafür, dass das Stück gem. § $28 \mathrm{Nr}$. 1 KGSG unrechtmäßig aus einem Vertragsstaat des UNESCO-Übereinkommens ausgeführt worden sei. Da neben den vom Beklagten behaupteten Ursprungsländern Myanmar und China jedenfalls auch Japan in Betracht komme und die Figur von dort rechtmäßig hätte ausgeführt werden können, seien die Voraussetzungen des $\S 32$ Abs. 2 KGSG nicht erfüllt, der analog auch auf § 28 Nr. 1 KGSG anzuwenden sei. Die (zugänglichen) Gesetze von China sähen die vom Beklagten zitierte Rechtsfolge nicht vor. Art. 2 Abs. 3 Kulturgutschutzgesetz der Volksrepublik China vom 19.11.1982, zuletzt geändert am 24.04.2015, enthalte für vor 1911 geschaffene Kulturgüter kein absolutes Ausfuhrverbot. Es bleibe unklar, auf welcher rechtlichen Basis der Beklagte von einem Verstoß bei einer möglichen Ausfuhr der Statue aus China ausgehe. Auch die Rechtslage in Myanmar verbiete eine Ausfuhr nicht. Eine Sicherstellung nach § 33 KGSG setze des Weiteren als ungeschriebenes Tatbestandsmerkmal ein tatsächlich bestehendes staatliches Sicherungsbedürfnis voraus. Dieses werde vom Beklagten nicht dargelegt. Die Sicherstellung könne auch nicht auf $\S 33$ Abs. 1 Nr. 1 (sic.) i.V.m. § 30 KGSG gestützt werden. § 30 KGSG setze voraus, dass es sich bei der streitgegenständlichen Figur um „nationales Kulturgut" eines Mitglieds- oder Vertragsstaats im Sinne des Kulturgutschutzgesetzes handele. Beides sei nicht der Fall. Die diesbezüglichen Behauptungen des Beklagten würden bestritten. Mit der Aufhebung des rechtswidrigen Verwaltungsakts werde auch die Aufhebung der mit ihm verbundenen Vollzugsfolgen beantragt.

Der Kläger beantragt, den undatierten Bescheid des Ministeriums für Wissenschaft, Forschung und Kunst BadenWürttemberg (Datum des in den Behördenakten enthaltenen Entwurfs: 12.06.2018), mit welcher die Sicherstellung der am 30.05.2018 vom Zollamt Heidelberg angehaltenen BuddhaStatue verfügt wurde, aufzuheben, den Beklagten zu verpflichten, diese Buddha-Statue (beschrieben unter Nummer 267 des Auktionskatalogs des Auktionshauses XXX, USA für die Versteigerung am 7. April 2018, 10 Uhr und wie in Nr. 2 des Klageantrags abgebildet) an den Kläger herauszugeben.

Der Beklagte beantragt, die Klage abzuweisen.

Zur Begründung trägt er vor: Der Kläger habe für die Behauptung, dass das Auktionshaus weder im Vorfeld der Auktion noch im Nachgang Nachweise zu Alter und Provenienz des Objekts erbracht habe, nicht dargelegt, inwieweit er diese überhaupt beim Auktionshaus angefordert habe. Er widerspreche sich insoweit selbst, indem er behaupte, dass das $\mathrm{Ob}$ jekt Gegenstand einer Nachlassauktion gewesen sei und eine Aussage zum letzten Verbleib des Objekts tätige, die jedenfalls dem Auktionskatalog so nicht entnommen werden könne. Das Objekt werde im Ausstellungskatalog durch "cracking ages" hinsichtlich des Alters näher spezifiziert. Dafür, dass es 
sich bei dem Objekt um eine japanische Arbeit handele, hat der Kläger keinerlei Beweise vorgelegt. Die Expertisen hätten allesamt das Objekt nicht nach Japan verortet. Im Rahmen der Zollanmeldung sei der Kläger von Herrn XXX vertreten worden, der gegenüber den Zollbeamten erklärt habe, dass das Ursprungsland des streitgegenständlichen Objekts vermutlich "Tibet" und dieses über 100 Jahre alt sei. Nach der Einschätzung von Dr. XXX, Direktorin des Museums für ostasiatische Kunst in XXX, sei die Provenienz der Statue nach China zu verorten und zeitlich der Ming Dynastie (ca. 15. - 16.Jahrhundert) zuzuordnen. Dem Gutachten von Herrn XXX zufolge, der das Objekt am 09.10.2018 in Augenschein genommen habe, sei der Buddha Shakyamuni aus China und der Ming-Dynastie zuzuordnen.

§ 33 Abs. 1 Nr. 1 b KGSG i.V.m. § 28 Nr. 1 KGSG sei verfassungsgemäß. Das Rechtsinstitut der Sicherstellung sei eine präventive Maßnahme zum Zweck der Gefahrenabwehr. Es solle verhindert werden, dass die Dauer der Ermittlung und der Prüfung des Sachverhaltes durch die zuständige Behörde genutzt werde, das Kulturgut dem staatlichen Zugriff zu entziehen. $\S 35$ ff. KGSG beinhalteten differenzierte Regelungen zur Aufhebung der Sicherstellung und der Entschädigung. $\S 33$ KGSG stelle eine verfassungsmäßige Inhalts- und Schrankenbestimmung des Art. 14 GG dar.

Die Voraussetzungen der Sicherstellung gemäß § 33 KGSG lägen vor. Es bestehe der hinreichende Verdacht, dass es sich bei der Buddha-Statue um Kulturgut handele, dessen Einfuhr gemäß § 28 Nr. 1 KGSG verboten sei, da China seit dem 28.11.1989 UNESCO-Vertragsstaat und die Ausfuhr von Kulturgut durch chinesische Rechtsvorschriften beschränkt sei. Im vorliegenden Fall handele es sich um eine Einfuhr über die USA als Drittstaat. Der "hinreichender Verdacht" bezüglich der unrechtmäßigen Ausfuhr eines Kulturguts gem. § 28 Nr. 1 KGSG liege vor. Die fachlichen Einschätzungen anhand der Digitalfotos des Objekts von Frau Dr. XXX genügten für die Begründung eines "hinreichenden" Verdachts der Echtheit, der Provenienz in China und der zeitlichen Einordnung in die Ming Dynastie. Die Inaugenscheinnahme durch Herrn XXX habe diesen Verdacht weiter erhärtet. Der hinreichende Verdacht bezüglich des zunächst ebenfalls in Frage kommenden Herkunftslands Myanmar habe sich nicht bestätigt. Das Objekt stelle gemäß Art. 2 des Kulturgutschutzgesetzes der Volksrepublik China Kulturgut dar. Aufgrund des Alters des Objekts sei dessen Ausfuhr aus China generell verboten. Nach schriftlichen Informationen der chinesischen Botschaft im Jahr 2016 (also nach der letztbekannten Gesetzesnovelle) an die Beauftragten für Kultur und Medien (BKM) bestehe in China ein Ausfuhrverbot für Kulturgüter, die vor 1911 geschaffen worden seien. Die in Gänze vorliegenden Gesetzesfassungen seit 1989 entsprächen einander in Bezug auf die relevanten Rechtsvorschriften zu Kulturgut (Art. 2 Abs. 3), Ausfuhr (Art. 60 ff.), und Kunsthandelsregulierungen (Art. 50 ff.). Die verschiedenen Fassungen der Gesetze seien für die Information auf dem Portal von Relevanz, um die Rechtslage bei Ausfuhr aus dem Herkunftsstaat entsprechend recherchieren zu können, und hilfreich, wenn - wie in China - die aktuellen Gesetze nur verschlüsselt einsehbar seien. Die UNESCO verfahre in ihrer Datenbank nicht anders. Ferner seien Skulpturen, namentlich auch Figuren aus Holz, vom Neolithikum bis 1949 in der von der ICOM herausgebrachten "roten Liste" von China aufgeführt. Die "rote Liste" enthalte Objekte mit Beispielen von Objekttypen und -kategorien, die besonders gefährdet seien, illegal gehandelt zu werden, weil sie Ausfuhrbeschränkungen oder Ausfuhrverboten unterlägen. Nach der Zielsetzung der roten Liste von ICOM sollten auch Kunst- und Antiquitätenhändler sowie Sammler in der roten Liste aufgeführte Kulturgüter nicht erwerben, ohne zunächst deren Ursprung überprüft zu haben. Obwohl der Buddha der "roten Liste“ unterfalle, sei der Kläger den Empfehlungen von ICOM nach derzeitigem Sachstand nicht nachgekommen. Entscheidend sei, dass die für den Kläger relevante Auskunft, dass chinesische Kulturgüter, die vor 1949 hergestellt würden, jedenfalls einer Ausfuhrgenehmigung bedürften oder gar einem Ausfuhrverbot unterfielen, über das Staatenportal einsehbar gewesen sei. Jedenfalls sei für das Objekt eine Ausfuhrgenehmigung gemäß Art. 60 des Kulturgutschutzgesetzes der Volksrepublik China erforderlich. Dem ebenfalls über das Staatenportal einsehbaren Kulturgutschutzgesetz der Volksrepublik China sei zudem zu entnehmen, dass eine Ausfuhrgenehmigung aus China erforderlich sei (Art. 60 ff), sofern die Objekte nicht wegen ihres Wertes und Alters bereits dem generellen Ausfuhrverbot unterlägen. Diese ausfuhrgenehmigungspflichtigen Objekte würden in Art. 8 der Verwaltungsreglungen für die Prüfung und Genehmigung des Im- und Exports von Kulturgut näher konkretisiert.

Die Buddha-Statue unterfalle unstreitig diesen Regelungen des chinesischen Kulturgutschutzgesetzes. China sei in Bezug auf den privaten Handel mit Kulturgütern (auch Verkäufe im Inland) und hierfür erforderliche Lizenzen und Nachweispflichten besonders rigide und sehe eine Ausfuhrgenehmigung für alle vor 1949 geschaffenen Kulturgüter vor. Ferner falle das Objekt auch unter die "rote Liste“. Daher könne für ein chinesisches Objekt erwartet werden, dass der Einführende gem. § 30 KGSG die Verkaufs-/Ausfuhr-/Zollunterlagen vorlege. Über Informationen zum Zeitpunkt der Verbringung des Objekts aus dem Herkunftsstaat, verfüge eher der Einführende, als die deutschen Behörden. Das Objekt in einem dritten Staat gekauft zu haben, entledige nicht von der Pflicht nach $\S 30$ KGSG, wenn aufgrund des Alters oder Wertes des Objektes anfängliche Hinweise bestünden, dass es sich um ein Objekt handeln könnte, welches von seinem Herkunftsstaat als nationales Kulturgut eingestuft werden könne. Hier erfordere die Einfuhr, wenn eine Beibringung von Zolldokumenten und Ausfuhrgenehmigung wegen der lange zurückliegenden Ausfuhr nicht möglich sei, jedenfalls nähere Provenienzangaben dazu, dass das Stück bereits vor In-Kraft-Treten des UNESCOAbkommens sich außerhalb seines Herkunftsstaates befunden habe. Der Einführende sei daher gehalten, zur Frage des Ausfuhrzeitpunktes bzw. zur Nichtanwendbarkeit des Einfuhr- 
verbots aus anderen Gründen Angaben zu machen, auf deren Basis entschieden werden könne, ob weiterhin von einem hinreichenden Verdacht nach $\S 33$ Absatz 1 Nr. 1 b bzw. Absatz 1 Nr. 2 KGSG auszugehen sei. Dem sei der Kläger bislang nicht nachgekommen. Der Beklagte trage keine Beweislast dafür, dass das Objekt schon vor dem 26.04.2007 in die USA gelangt sei. Vielmehr obliege es dem Kläger, anhand geeigneter Unterlagen darzulegen, dass das Objekt den Herkunftsstaat vor diesem Stichtag verlassen habe. Die Sichersteilung diene unter anderem der Sicherung von Rückgabeverfahren nach §§ $49 \mathrm{ff}$. KGSG. Insofern sei die in $§ 52$ Abs. 2 KGSG geregelte Beweislastumkehr nach Sinn und Zweck der Vorschrift auch im Rahmen der Sicherstellung anzuwenden. Vor dem Hintergrund der in § 30 KGSG geregelten Nachweispflicht des Einführenden könne nichts anderes gelten. Gem. § 30 KGSG habe der Einführende den Nachweis der Rechtmäßigkeit zu erbringen. $\S 30$ KGSG begründe eine gesetzliche Beweislastregel zu Lasten des Einführenden; der Einführer müsse darlegen, dass das Objekt ausgeführt worden sei, als es noch nicht unter Schutz gestanden habe. Gem. § 30 KGSG müsse der Kläger den Nachweis der Rechtmäßigkeit erbringen, was bisher nicht erfolgt ist, weshalb eine unrechtmäßige Ausfuhr gem. § 28 Nr. 1 KGSG jedenfalls nicht ausgeschlossen werden könne.

Die Sicherstellung sei auch wegen der fehlerhaften Dokumentation gem. § 30 KGSG rechtmäßig. Gem. § 33 Abs. 1 Nr. 2 KGSG i. V. m. § 30 KGSG habe die zuständige Behörde Kulturgut sicherzustellen, wenn bei der Einfuhr die nach § 30 KGSG erforderlichen Unterlagen nicht vorgelegt würden. Gem. § 30 KGSG habe, wer Kulturgut einführe, sofern es von einem Vertragsstaat als nationales Kulturgut eingestuft oder definiert worden sei, zum Nachweis der Rechtmäßigkeit der Ausfuhr aus dem Herkunftsstaat im Sinne von § 28 Nr. 1 KGSG entsprechende Unterlagen mitzuführen. Von Seiten des Klägers sei bisher kein entsprechender Nachweis der Rechtmäßigkeit der Einfuhr erbracht worden. § 30 KGSG begründe eine Beweislastregel zu Lasten des Einführenden; dieser müsse darlegen, dass das Objekt ausgeführt worden sei, als es noch nicht unter Schutz gestanden habe bzw. es müsse eine Ausfuhrgenehmigung vorgelegt werden. Obwohl der Vertreter des Klägers gegenüber dem Zoll Tibet, d.h. China, als vermutliches Ursprungsland angegeben habe, seien bislang keine entsprechenden Ausfuhrunterlagen gemäß Art. 61 des chinesischen Kulturgutgesetzes vorgelegt worden.

In seiner Replik hat der Kläger erwidert: Die vom Beklagten zitierten ICOM-Vorschriften würden vom „International Council of Museums" herausgegeben, richteten sich an Museen und hätten keine Rechtsqualität. Hieraus könne keine "Sorgfaltspflichtverletzung" hergeleitet werden. Mit der Vorlage der Auskunft des Auktionshauses sei hinreichend nachgewiesen, dass die streitgegenständliche Statue vor dem entsprechenden Stichtag aus China ausgeführt worden sei. Das Fehlen einer Ausfuhrgenehmigung könne auch dafürsprechen, dass die Statue zu einem Zeitpunkt ausgeführt worden sei, als solche Genehmigungen noch nicht benötigt worden seien. Hierfür spreche - die Behauptungen der Beklagten als wahr unterstellt - das Alter des Objekts. Bei einem Objekt, das 500 Jahre alt sei, gebe es einen sehr viel größeren Zeitraum, in welchem dieses das Gebiet der Volksrepublik China legal hätte verlassen können. Mit China bestünden seit mehreren Jahrhunderten umfangreiche Handels- und Geschäftsbeziehungen und auf diesen Routen hätten mit Sicherheit auch zahlreiche Buddha-Statuen (legal) das Land verlassen. Die Volksrepublik China sei seit dem 26.04.2007 ein autokratisch geführter Polizeistaat gewesen. Angesichts der dortigen drakonischen Strafen sei es sehr unwahrscheinlich, dass jemand eine geschützte Statue ausführe und in die USA verbringe, nur um sie 10 Jahre später bei einer Varia Auktion für einen knapp vierstelligen Betrag zu versteigern. Die vom Beklagten als „Beweislastumkehr" verstandene Regelung des § 52 Abs. 2 S. 1 KGSG sei verfassungswidrig. Der deutsche Gesetzgeber sei bei seiner Formulierung weit über das hinausgegangen, was durch das UNESCO-Übereinkommen von den Vertragsstaaten gefordert werde, ohne diesen Eingriff hinreichend zu rechtfertigen. Es gebe zahlreiche Sachverhaltsvarianten, bei denen der Eigentümer eines Kulturguts aus tatsächlichen, aber rechtmäßigen Gründen gehindert sei, den entsprechenden Nachweis zu führen. Die „Beweislastumkehr" führe hier zu einer unverhältnismäßigen Beschneidung seiner prozessualen Rechte mit der Folge eines Eingriffs in Art. 14 GG.

Dem Gericht liegen die Akten des Beklagten vor. Auf diese sowie auf den Inhalt der zwischen den Beteiligten gewechselten Schriftsätze wird ergänzend Bezug genommen.

\section{Entscheidungsgründe}

Die Klage ist zulässig und begründet.

Der angefochtene Bescheid des Ministeriums für Wissenschaft, Forschung und Kunst Baden-Württemberg ist rechtswidrig und verletzt den Kläger daher in seinen Rechten ( $§ 113$ Abs. 1 Satz 1 VwGO).

Die Voraussetzungen des § 33 Abs. 1 KGSG, welcher die zuständigen Behörden ermächtigt, "Kulturgut" sicherzustellen, d.h. einen staatlichen Gewahrsam zu begründen, liegen nicht vor.

Maßgeblicher Zeitpunkt für die Beurteilung der Sach- und Rechtslage ist die mündliche Verhandlung.

Die Rechtmäßigkeit eines mit einer Anfechtungsklage angegriffenen Verwaltungsakts beurteilt sich zwar in vielen Fällen nach der Sach- und Rechtslage im Zeitpunkt der letzten Behördenentscheidung. Maßgeblich für die Bestimmung des entscheidungserheblichen Beurteilungszeitpunkts ist allerdings nicht das Prozessrecht, sondern das jeweilige materielle Recht (VGH Bad.-Württ., Urt. v. 09.04.2019 - 1 S 1813/17 - juris, Rn 46 ff.). Wendet sich ein Kläger - wie hier - im Wege der Anfechtungsklage gegen eine auf $\S 33$ KGSG gestützte Sicherstellungsverfügung, beurteilt sich deren Rechtmäßig- 
keit nach der Sach- und Rechtslage im Zeitpunkt der letzten mündlichen Verhandlung. Das ergibt sich aus den materiellrechtlichen Vorgaben des $\S 35$ KGSG, der die Modalitäten regelt, unter denen die Sicherstellung wieder aufzuheben ist. So ist dies u.a. dann der Fall, wenn der hinreichende Verdacht nach $\S 33$ Abs. 1 Nr. 1 KGSG entfallen ist ( 35 Abs. 1 Nr. 1 KGSG) oder wenn im Falle des § 33 Abs. 1 Nr. 1 b KGSG die Voraussetzungen des Rückgabeanspruchs nach Kapitel 5 KGSG nicht vorliegen (§ 35 Abs. 1 Nr. 3 a KGSG). Hieraus folgt, dass die Behörde verpflichtet ist, nach der einmal erfolgten Sicherstellung fortlaufend zu prüfen, ob die Sicherstellung weiterhin gerechtfertigt ist. Hat sie die Sicherstellung als Verwaltungsakt verfügt, hat sie diesen Verwaltungsakt also unter Kontrolle zu halten. Das bedeutet zum einen, dass die Sicherstellung ein Verwaltungsakt mit Dauerwirkung ist. Aus dieser materiellen Rechtslage folgt zum anderen, dass maßgeblicher Zeitpunkt für die Überprüfung der Rechtmäßigkeit einer Sicherstellung, die - wie hier - noch andauert, im Rahmen der Anfechtungsklage der Zeitpunkt der Entscheidung des Gerichts ist (vgl. für den Fall der Sicherstellung nach $\S 32$ Abs. 1 PolG, die mit der Aufhebungsvorschrift des $\S 32$ Abs. 4 PolG ähnlich wie die Sicherstellung nach dem KGSG geregelt ist: VGH Bad.-Württ., Urt. v. 09.04.2019 - 1 S 1813/17 - juris, Rn. 47).

Nach § 33 Abs. 1 KGSG hat die zuständige Behörde Kulturgut sicherzustellen, wenn der hinreichende Verdacht besteht, dass es a) entgegen einem Verbot nach $\S 21$ ausgeführt werden soll oder b) entgegen einem Verbot nach $\S 28$ eingeführt worden ist ( 33 Abs. 1 Nr. 1 KGSG) oder wenn bei der Einfuhr die nach $\S 30$ KGSG erforderlichen Unterlagen nicht vorgelegt werden (§ 33 Abs. 1 Nr. 2 KGSG).

Bei der sichergestellten Buddha-Statue handelt es sich zwar um "Kulturgut" im Sinne der Legaldefinition des § 2 Abs. 1 Nr. 10 KGSG. Danach ist "Kulturgut" jede bewegliche Sache oder Sachgesamtheit von künstlerischem, geschichtlichem oder archäologischem Wert oder aus anderen Bereichen des kulturellen Erbes, insbesondere von paläontologischem, ethnographischem, numismatischem oder wissenschaftlichem Wert. Nach diesem weiten Begriff (vgl. dazu: Elmenhorst/Wiese, KGSG, 2018, § 2 Rn. 13), handelt es sich bei der BuddhaStatue, als Sache, die von den damit befassten sachkundigen Personen übereinstimmend als Original angesehen worden ist, um einen Gegenstand, der zumindest von künstlerischem Wert ist und damit einen Wert aus dem Bereich des - ganz umfassend zu verstehenden - kulturellen Erbes aufweist.

Allerdings liegen hier weder die Voraussetzungen für den hier in Betracht kommenden Sicherstellungstatbestand des $\S 33$ Abs. 1 Nr. 1 b KGSG (1.) noch diejenigen des § 33 Abs. 1 Nr. 2 KGSG (2.) vor.

1. Gem. § 33 Abs. 1 Nr. 1 b KGSG ist eine Sicherstellung nur dann zulässig, wenn der hinreichende Verdacht besteht, dass die Einfuhr des Kulturguts entgegen einem Verbot nach $\S 28$ KGSG erfolgt ist. Nach der Bestimmung des hier allein in Be- tracht kommenden $\S 28 \mathrm{Nr} .1 \mathrm{KGSG}$ ist die Einfuhr von Kulturgut verboten, wenn es von einem Mitgliedstaat oder Vertragsstaat als nationales Kulturgut eingestuft oder definiert worden ist und unter Verstoß gegen dessen Rechtsvorschriften zum Schutz nationalen Kulturgutes aus dessen Hoheitsgebiet verbracht worden ist. Als Vertragsstaat ist - in Abgrenzung zu den Mitgliedstaaten der Europäischen Union - jeder andere Staat definiert, für den das UNESCO-Übereinkommen, d. h. das Übereinkommen über Maßnahmen zum Verbot und zur Verhütung der rechtswidrigen Einfuhr, Ausfuhr und Übereignung von Kulturgut (BGBI. 2007 II, S. 626 f.; vgl. § 2 Abs. 1 Nr. 17 KGSG) bindend ist (§ 2 Abs. 1 Nr. 19 KGSG).

Es liegt hier kein Sachverhalt vor, welcher geeignet wäre, einen hinreichenden Verdacht zu begründen, dass die Voraussetzungen des $\S 28$ Abs. 1 KGSG vorliegen. Letzteres hängt davon ab, woher die Buddha-Statue stammt (a), wann diese aus dem etwaigen Vertragsstaat gelangte (b) und ob - ggf. welche rechtlichen Vorgaben für eine Ausfuhr zu diesem Zeitpunkt bestanden, wobei auch das Alter der Statue eine Rolle spielt (c). Zumindest hinsichtlich der letzten beiden Punkte bestehen Unklarheiten, die der Annahme eines hinreichenden Tatverdachts entgegenstehen (d).

a. Hinsichtlich des hier maßgeblichen Vertragsstaats stellt der Beklagte auf die Volksrepublik China ab, welche seit dem 28.11.1989 UNESCO-Vertragsstaat ist. Soweit ersichtlich, dürfte die Statue - sofern sie aus China stammt - auch dort als Kulturgut eingestuft werden. Hinsichtlich der heranzuziehenden Rechtsgrundlage besteht jedoch die Problematik, dass - wie sich aus dem aufgrund der Bestimmung des § 4 KGSG aufzubauenden Internetportal der Bundesregierung ergibt - die chinesischen Gesetzestexte nicht durchgängig und nicht vollständig zugänglich sind (www.kulturgutschutz-deutschland.de). Nach den Ausführungen des Internetportals soll ein weiter Kulturgutsbegriff gelten und wertvolle Kunstwerke und Objekte des Kunsthandwerks aus verschiedensten geschichtlichen Epochen dazugehören. Entsprechendes lässt sich auch dem Link auf den englischen Auszug des Gesetzestextes der Law of the People's Republic of China on the Protection of Cultural Relics v. 19.11.1982 (letzte Änderung 24.04.2015, s. hk.lexiscn.com) entnehmen, wonach die Buddha-Statue wohl als Kulturgut im Sinne des Art. 2 Nr. 3 der genannten Vorschrift einzuordnen sein dürfte. Der Kläger bestreitet jedoch die Herkunft der Statue. Ob die hier vorliegenden sachkundigen Äußerungen einen hinreichenden Schluss auf die chinesische Abstammung der Statue zulassen, kann offenbleiben. Selbst wenn man die chinesische Herkunft unterstellen würde, kann die zum Zeitpunkt der mündlichen Verhandlung bestehende Sachlage aus anderen Gründen keinen hinreichend Verdacht dafür begründen, dass die Statue unter Verstoß gegen Chinas Rechtsvorschriften zum Schutz nationalen Kulturgutes aus dessen Hoheitsgebiet gelangt ist. 
b. Es bestehen bereits keine verlässlichen Anhaltspunkte, die einen Schluss auf den Zeitpunkt zuließen, zu dem die BuddhaStatue aus ihrem Herkunftsland verbracht worden ist. Hiervon abhängig ist die dann zu beantwortende Frage, ob im Herkunftsland zu diesem Zeitpunkt überhaupt Ausfuhrbeschränkungen bestanden haben und - ggf. - wie diese lauteten.

Fest steht nur, dass die Statue nicht direkt von China aus, sondern über die USA nach Deutschland gelangt ist. Der Kläger hat die Statue am 07.04.2018 bei einer Internetauktion eines US-amerikanischen Auktionshauses ersteigert, und diese ist von den USA aus nach Deutschland versandt worden. Des Weiteren bietet die E-Mail des Auktionshauses vom 29.10.2020, wonach die Statue aus einem Nachlass stammen soll, einen Anhalt dafür, dass diese sich zuvor in den USA auch bereits geraume Zeit befunden hat. Insoweit wird in der E-Mail ausgeführt, dass dies bereits vor dem 06.04.2007 der Fall gewesen sein soll.

Diese magere Tatsachengrundlage ist jedoch nicht geeignet, einen hinreichenden Grund für die Annahme zu bilden, die Buddha-Statue habe China zu einem Zeitpunkt verlassen, in dem ein für derartige Kulturgüter bestehendes Ausfuhrverbot bereits gegolten hat.

(1.) Dies folgt bereits daraus, dass nicht hinreichend gerichtsverwertbar gesichert ist, wie sich die derzeitige Rechtslage in China darstellt.

Nach dem vorerwähnten Internetportal der Bundesregierung gibt es in China folgende Rechtsgrundlagen:

1. Kapitel VI, Kulturgutschutzgesetz der Volksrepublik China vom 19.11.1982 (Law of the People's Republic of China on the Protection of Cultural Relics) zuletzt geändert am 24. April 2015 (Law of the People's Republic of China on the Protection of Cultural Relics). Das Gesetz ist lizenzfrei lediglich in der Gesetzesfassung aus 2002 verfügbar auf dem chinesischen, englischsprachigen Informationsportal. In der LexisNexis Datenbank ist die aktuelle Gesetzesfassung nur eingeschränkt verfügbar. Bislang ebenfalls noch eingeschränkt verfügbar ist der aktuelle Gesetzestext aber mit umfassenden Links zu den Gesetzesmaterialien auf der Informationsplattform open repository on cultural property

2. Kapitel VI der Durchführungsverordnung für das Kulturgutschutzgesetz der Volksrepublik China (Regulation for the Implementation of the Law of the People's Republic of China on the Protection of Cultural Relics), lizenzfrei in der Fassung aus 2003, in der aktuellen Fassung vom 7. Dezember 2013 nur eingeschränkt verfügbar

3. Verwaltungsvorschriften für die Prüfung und Genehmigung des Im- und Exports von Kulturgut, herausgegeben vom Kulturministerium der Volksrepublik China (Rules for the Review and Approval of Entry and Exit of Cultural Objects, issued by the Ministry of Culture of the People's Republic of China)

4. Standards der Prüfung und Genehmigung des Exports von Kulturgut (Standards for the Review and Approval of Exit of Cultural Objects)

5. Verwaltungsvorschriften für die Ausstellung von Kulturgut im Ausland (Administrative Rules for Outbound Exhibitions of Cultural Objects)

Einen vollständigen Zugriff auf diese Vorschriften gibt es nicht. Vielmehr wird in dem Internetportal ausgeführt, dass die aktuellen Gesetzesfassungen nicht in Gänze frei einsehbar sind. Das Portal bezieht sich insoweit lediglich auf - nicht näher spezifizierte - direkte Rechtsauskünfte von Staatsseite und listet die Gegenstände auf, für die ein absolutes Ausfuhrverbot bestehen soll, nämlich für:

- Kulturgut, das vor 1911 (einschließlich) produziert oder geschaffen wurde

- Charakteristisches Kulturgut ethnischer Minderheiten, welches vor 1966 (einschließlich) produziert oder geschaffen wurde

- Kulturgut innerhalb der Kategorien, die festgelegt werden von den Standards für die Prüfung und Genehmigung des Exports von Kulturgut

- Werke, die festgelegt werden von den Bestimmungsstandards für beschränkt exportierbare Werke berühmter Kalligraphen und Maler, die nach 1949 verstorben sind und berühmter Kalligraphen und Maler, die zwischen 1795 und 1949 gelebt haben

- Werke, die festgelegt werden von den Bestimmungsstandards für beschränkt exportierbare Werke berühmter Kalligraphen und Maler, die nach 1949 verstorben sind (2. Charge)

- Werke, die einzigartig oder transportempfindlich/ zerbrechlich sind und offiziell als Klasse-1-Objekte identifiziert wurden (Art. 62 des chinesischen Kulturgutschutzgesetzes)

- die zuvor niemals offiziell in China ausgestellt worden sind (Art. 49 der Durchführungsverordnung für das chinesische Kulturgutschutzgesetz)

- die auf der ersten, zweiten oder dritten Liste solcher Objekte verzeichnet ist, welche nicht im Ausland ausgestellt werden dürfen 
Des Weiteren listet das Internetportal das Kulturgut auf, bei dem die Ausfuhr unter Genehmigungsvorbehalt stehen soll, nämlich Kulturgut, welches vor 1949 (einschließlich) produziert oder geschaffen wurde und von einigem geschichtlichen, künstlerischen oder wissenschaftlichen Wert sei und die nach den Verwaltungsvorschriften für die Prüfung und Genehmigung des Im- und Exports von Kulturgut in folgende Kategorien eingeteilt seien:

- Kunstwerke und Objekte des Kunsthandwerks, die vor 1949 geschaffen wurden

- Manuskriptoriginale und eigenhändige Manuskripte, Literaturdokumente, Bücher und Referenzmaterial deren Geschichte ab 1949 oder früher begann

- Fassbare kulturelle Zeugnisse gesellschaftlicher Systeme, gesellschaftlicher Produktion und des gesellschaftlichen Lebens verschiedenster Nationalitäten, die mindestens seit 1949 bestehen

- Charakteristische, gegenständliche Kulturgüter, die mit bedeutenden geschichtlichen Ereignissen nach 1949 oder mit berühmten Persönlichkeiten verbunden sind

- Charakteristische Gegenstände, die mit Produktionsaktivitäten, Gepflogenheiten und Bräuchen, Kultur, Kunst und religiösen Anschauungen verschiedenster Nationalitäten nach 1949 verbunden sind

- Werke verstorbener bekannter Maler und Kunsthandwerker, deren Ausfuhr von der State Administration of Cultural Heritage beschränkt wurde

- Prähistorische Fossilien von Affen, Menschen sowie von Wirbeltieren des Quartärs, die mit menschlichen Aktivitäten im Zusammenhang stehen

Ohne Verifizierung und der Nachprüfung zugängliche Angaben der Quellen, aus denen sich die Zusammenfassung im Internetportal herleitet, lässt sich jedoch nicht verlässlich die in China bestehende Rechtslage im Hinblick auf die dortigen Ausfuhrbeschränkungen beurteilen.

(2.) Aber auch selbst dann, wenn man - wie im Internetportal dargestellt - annähme, dass eine gegenwärtig aus China ausgeführte Buddha-Statue in der hier streitgegenständlichen Art einem Ausfuhrverbot unterliegt, so ist dieser Umstand nicht geeignet, einen Schluss darauf zuzulassen, dies sei auch im hier zugrunde zulegenden Zeitpunkt der Ausfuhr der Fall gewesen. Denn die im Internetportal enthaltenen Informationen, welche sich auf das Kulturgutschutzgesetz der Volksrepublik China vom 19.11.1982 beziehen, das zuletzt am 24.04.2015 geändert wurde, lassen lediglich auf die gegenwärtige Rechtslage schließen. Eine hinreichende Tatsachengrundlage für die Annahme, dass die Buddha-Statue erst unter Geltung der aktuellen Rechtslage aus China ausgeführt wurde, ist jedoch nicht vorhanden. Es fehlen Erkenntnisse dazu, dass inhaltsgleiche Rechtsvorschriften bereits zuvor fortwährend seit Schaffung der Buddha-Statue gegolten haben oder zumindest, dass ein Ausfuhrverbot bestanden hat. Demgegenüber bestehen durchaus Anhaltspunkte, dass in Vorgängervorschriften weniger strenge Regelungen galten, insbesondere, dass die Ausfuhr von Kulturgut erst dann verboten war, wenn es zu einem weitaus früheren Zeitpunkt produziert worden war. Nach german.china org.cn (Stand 21.09.2007) hatte damals das Chinesische Zollbüro eine neue Regelung zur erlaubten Ausfuhr von einheimischen Kulturgütern ausgearbeitet, welche das Spektrum der zur Ausfuhr verbotenen Gegenstände erweitert habe. Gegenstände, die vor 1949 hergestellt worden seien, hätten nicht mehr aus China ausgeführt werden dürfen. Zuvor habe die Regel gegolten, dass keine Gegenstände, die vor 1795 (letztes Herrschaftsjahr des Qing-Kaisers Qianlong) produziert worden seien, das Land hätten verlassen dürfen.

c. In Abhängigkeit dazu, dass eine hinreichend gesicherte Kenntnis erforderlich ist, wann welche Rechtsgrundlage für ein Ausfuhrverbot in China gegolten hat, fehlt - als Voraussetzung für die Prüfung, welche Rechtsgrundlage anwendbar ist - eine hinreichende Tatsachengrundlage, welche sowohl einen verlässlichen Rückschluss auf das Alter der Buddha-Statue als auch auf den Zeitpunkt der Verbringung aus China zuließe.

Zum Alter der Statue wurden vom Beklagten verschiedene Stellungnahmen fachkundiger Personen eingeholt, welche dieses keinesfalls einheitlich angeben. Vielmehr liegt die Spannbreite zwischen dem 15. und dem 19. Jahrhundert: In einer ersten Einschätzung von Frau XXX und Herrn XXX vom XXX (E-Mail vom 30.05.2018) ist davon die Rede, dass diese aus dem 17./18. Jahrhundert stammt. Herr XXX, Kurator für asiatische Kunst, Stadt XXX datiert die Statue ins 18./19. Jahrhundert und gibt an, dass sie keinen allzu hohen Wert hat (E-Mail vom 25.06.2018). Herr XXX ordnet den Buddha in seiner Äußerung vom 14.10.2018 der Ming-Dynastie, 15./16. Jahrhundert, zu und gibt den mittleren Marktwert mit 6.000 bis $10.000 €$ an. Frau Dr. XXX von der Stadt XXX datiert das Alter des Buddha ebenso und führt aus: Die Figur habe provinziellen Charakter. Falls sie schon vor den 1970er Jahren Teil einer westlichen Sammlung gewesen sei, sehe sie keine Probleme im Hinblick auf das Kulturgüterschutzgesetz.

d. Die voranstehend aufgezeigten Unklarheiten, welche Zeitpunkte für die Herstellung und die Verbringung der Buddha-Statue aus dem Herkunftsland zugrunde zu legen sind und - abhängig davon - welche Rechtsgrundlage damals galt, stehen der Annahme eines hinreichenden Tatverdachts entgegen, dass die Einfuhr entgegen einem Verbot nach § 28 KGSG erfolgt ist.

Das Kulturgutschutzgesetz enthält keine Definition des Begriffs des hinreichenden Tatverdachts. Insoweit forderte das Vorgängergesetz, das KultGüRückG 2007, in § 7 Abs. 2 Satz 1 
und $\S 8$ Abs. 2 Satz 1 noch einen dringenden Verdacht. Unter welchen Voraussetzungen ein hinreichender Tatverdacht vorliegt, bestimmt sich mangels eigener Definition im KGSG nach den entsprechend heranzuziehenden Grundsätzen der Strafprozessordnung, nämlich denjenigen zu § 170 Abs. 2 StPO. Dies entspricht dem bisherigen Definitionsverständnis in §§ 7 Abs. 2 Satz 1 und 8 Abs. 2 Satz 1 KultGüRückG 2007. Die Anklage wird gemäß § 170 Abs. 1 StPO nur erhoben, wenn gegen den Beschuldigten ein hinreichender Tatverdacht besteht, anderenfalls stellt die Staatsanwaltschaft das Verfahren gem. $\S 170$ Abs. 2 StPO ein. Hinreichender Tatverdacht ist gegeben, wenn nach vorläufiger Bewertung des sich aus dem gesamten Akteninhalt ergebenen Sachverhalts und der Beweisergebnisse eine Verurteilung des Beschuldigten wahrscheinlicher als ein Freispruch ist, mithin eine überwiegende Wahrscheinlichkeit für eine Verurteilung besteht (BeckOK StPO/Gorf, 36. Ed. 01.01.2020, StPO $\S 170$ Rn. 2, m.w.N.). Übertragen auf die Anordnung der Sicherstellung nach $\S 33$ Abs. 1 Nr. 1 b heißt dies, dass es für die Annahme eines hinreichenden Tatverdachts genügt (aber auch erforderlich ist), dass dessen Tatbestandsvoraussetzungen überwiegend wahrscheinlich vorliegen (Elmenhorst/Wiese, KGSG 2018, § 35 Rn 18).

Eine solche überwiegende Wahrscheinlichkeit, dass die Einfuhr der Buddha-Statue entgegen einem Verbot nach § 28 KGSG erfolgt ist, besteht hier aus den vorgenannten Gründen nicht. Es steht fest, dass der Buddha nicht direkt aus China importiert, sondern in den USA ersteigert worden ist. Lt. E-Mail des Auktionshauses soll er sich bereits vor dem 06.04.2007 in den USA befunden haben. Demzufolge ist das Verbringungsdatum in die USA offen und damit auch, welche chinesischen Vorschriften Anwendung finden, die zudem nicht ohne weiteres zugänglich sind. Nach dieser Sachlage ist es zumindest ebenso wahrscheinlich, dass die Buddha-Statue China zu einer Zeit verlassen hat, in der keine Ausfuhrbeschränkungen bestanden. Insoweit hat der Kläger zu Recht auf China als alte Handelsnation verwiesen. Sollte die Buddha-Statue tatsächlich aus der Ming-Dynastie (15./16. Jhdt.) stammen, ist ein Verlauf, dass sie China auf den damaligen Handelswegen bereits frühzeitig verlassen hat, zumindest ebenso wahrscheinlich wie ein solcher, dass sie erst später entgegen geltender Vorschriften aus China verbracht wurde. Zudem ist zu beachten, dass die USA schon immer Einwanderungsland gewesen sind. Die Geschichte der Chinesen in den Vereinigten Staaten von Amerika beginnt um die Mitte des 19. Jahrhunderts mit der Aufnahme der chinesisch-amerikanischen Seehandelsbeziehungen. Nachdem zunächst nur Kaufleute und Studenten Amerika bereist hatten, führte der kalifornische Goldrausch Mitte des 19. Jahrhunderts zum ersten großen chinesischamerikanischen Migrationsschub (Wikipedia: Geschichte der Chinesen in den Vereinigten Staaten). Mithin besteht auch eine Wahrscheinlichkeit dafür, dass die Statue bereits zu dieser Zeit über einen eingewanderten Chinesen in die USA kam.
Nach alledem ist zumindest offen, ob es zum Zeitpunkt, in dem die Buddha-Statue außer Landes gebracht wurde, Ausfuhrbestimmungen gab und wenn ja, welche. Daher kann von einer überwiegenden Wahrscheinlichkeit dafür, dass sie entgegen einem Verbot nach § 28 KGSG ausgeführt wurde, nicht die Rede sein und die Voraussetzungen für eine Sicherstellung nach § 33 Abs. 1 Nr. 1 b KGSG liegen nicht vor.

Eine Umkehr der Beweislast kommt bei einer solchen Fallgestaltung nicht in Betracht. Dies verbietet bereits der Wortlaut des $\S 33$ Abs. 1 Nr. 1 b KGSG, der ausdrücklich darauf abstellt, dass ein hinreichenden Tatverdacht vorliegen muss, dass die Ausfuhr entgegen einem Verbot nach § 28 KGSG erfolgt ist, die Tatbestandsvoraussetzungen also mit überwiegender Wahrscheinlichkeit vorliegen müssen. Lässt sich eine solche - wie hier - nicht feststellen, besteht kein Raum für eine Beweislastentscheidung, vielmehr sind die Voraussetzungen für eine Sicherstellung nicht erfüllt.

Auf die vom Kläger aufgeworfenen Fragen zur Verfassungsgemäßheit der Regelungen zur Sicherstellung nach $\S 33$ Abs. 1 Nr. 1 b KGSG kommt es daher nicht an.

2. Die Sicherstellung der Buddha-Statue lässt sich entgegen der Auffassung des Beklagten auch nicht auf $\S 33$ Abs. 1 Nr. 2 KGSG stützen.

Danach ist ein Kulturgut sicherzustellen, wenn bei der Einfuhr die nach § 30 KGSG erforderlichen Unterlagen nicht vorgelegt werden. Nach § 30 KGSG hat, wer Kulturgut einführt, sofern es von einem Mitgliedstaat oder Vertragsstaat als nationales Kulturgut eingestuft oder definiert worden ist, zum Nachweis der Rechtmäßigkeit der Ausfuhr aus dem Herkunftsstaat im Sinne von § 28 Nr. 1 KGSG entsprechende Unterlagen mitzuführen. Ein solcher Nachweis sind Ausfuhrgenehmigungen des Herkunftsstaates sowie sonstige Bestätigungen des Herkunftsstaates, dass das Kulturgut rechtmäßig ausgeführt werden konnte.

§ 30 KGSG knüpft an das Einfuhrverbot des § 28 KGSG an, indem es die Verpflichtung regelt, die rechtmäßige Ausfuhr von Kulturgut aus einem Mitglied- oder Vertragsstaat nachzuweisen. Geeignete Unterlagen sind insbesondere Ausfuhrgenehmigungen des Herkunftsstaates, sofern solche nach dessen Recht erforderlich sind. Nach Artikel 6 Buchstabe a des UNESCO-Übereinkommens sind Vertragsstaaten verpflichtet "eine geeignete Bescheinigung einzuführen, durch die der ausführende Staat bescheinigt, dass die Ausfuhr des betreffenden Kulturguts genehmigt ist. Jedes ausgeführte Kulturgut muss von einer solchen Bescheinigung begleitet werden“ (BTDrs. 18/7456, S. 91; Elmenhorst/Wiese, KGSG 2018, § 30 Rn. 3). Bei der Rechtmäßigkeit der Ausfuhr aus einem Mitglied- oder Vertragsstaat, ist nicht allein auf den letzten Aufenthaltsort des Kulturgutes außerhalb des Bundesgebietes abzustellen, sondern auf den Staat, aus dem das Kulturgut nach Würdigung der Gesamtumstände verbracht wurde und nach des- 
sen Regelungen es geschützt ist (BT-Drs. 18/7456, S. 91; Elmenhorst/Wiese, KGSG 2018, § 30 Rn. 4). Nach der Intention des Gesetzgebers soll durch § 30 KGSG vor allem die Einfuhr von Kulturgütern, die aus Raubgrabungen stammen, bzw. die Einfuhr von gestohlenem Kulturgut verhindert werden (BT-Drs. 18/7456, S. 91; s. auch Handreichung zum KSGS zu § 30).

Mit Blick darauf, dass $\S 33$ Abs. 1 Nr. 2 KGSG von „erforderlichen" Unterlagen spricht, ist es jedoch denknotwendig Voraussetzung, dass der Vertragsstaat im Zeitpunkt der Ausfuhr solche Bescheinigungen bereits eingeführt hatte. Hierfür spricht auch die bereits zitierte Vorschrift des Art. 6 Buchst. a des UNESCO-Übereinkommens. Denn bei einem Kulturgut, das etwa lange vor Existenz des UNESCO-Übereinkommens aus einem Herkunftsstaat verbracht worden ist, kann es derartige Bescheinigungen nicht geben.

Vorliegend ist die Buddha-Statue nicht direkt aus dem Herkunftsstaat, sondern aus den USA, die seit dem 02.09.1983 Vertragsstaat des UNESCO-Übereinkommens sind, eingeführt worden. Die USA stellen keine Ausfuhrgenehmigungen aus und sehen grundsätzlich auch keine Ausfuhrkontrolle für Kulturgüter vor (www.kulturgutschutz-deutschland.de).

Ob eine Ausfuhrgenehmigung aus dem Herkunftsland der Buddha-Statue notwendig war (bzw. ob es ein Ausfuhrverbot gab und daher keine entsprechenden Bescheinigungen ausgestellt wurden), hängt von ihrer Herkunft und Alter ab, des Weiteren davon, wann sie aus dem Herkunftsstaat verbracht worden ist und nach welcher damaligen Rechtslage sich die Ausfuhr beurteilte. Dies alles ist vorliegend jedoch offen.

Fehlen wie hier spezielle gesetzliche Beweislastregeln, richtet sich die Frage der materiellen Beweislast bzw. objektiven Feststellungslast nach dem Normbegünstigungsprinzip. Beweisbelastet ist danach derjenige, der aus einer unerweislichen Tatsache eine für ihn günstige Rechtsfolge ableiten möchte (VGH Bad.-Württ., Beschl. v. 29.03.2019 - 10 S 2788/17 - Rn. 13, juris). Die materielle Beweislast für die Tatsachen, die nach der zugrundeliegenden Norm Voraussetzung für die durch den Verwaltungsakt angeordnete Rechtsfolge sind, trägt im Rahmen der Eingriffsverwaltung stets die Behörde (Bayer. VGH, Beschl. v. 17.02.2020 - 12 CS 19.2505 - juris; Sodan/Ziekow, VwGO, 5. Aufl. 2018, § 108 Rn. 123 m.w.N.). Damit ist für das Vorliegen der oben genannten Umstände der Beklagte darlegungs- und beweispflichtig; er allein trägt insoweit die Darlegungs- und Beweis- bzw. Feststellungslast für den Sachverhalt, aus dem sich das Vorliegen der Voraussetzungen für das Einschreiten - vorliegend die Sicherstellung - ergeben soll. Ein Nachweis der Voraussetzungen ist dem Beklagten nicht gelungen.

Entgegen der Auffassung des Beklagten kann aus der Regelung des $\S 52$ KGSG, welcher den Rückgabeanspruch eines Vertragsstaates betrifft, keine Beweislastumkehr hergeleitet werden. Gem. § 52 Abs. 1 KGSG ist auf Ersuchen eines Ver- tragsstaates Kulturgut zurückzugeben, wenn es u.a. aus dessen Hoheitsgebiet nach dem 26.04.2007 unter Verstoß gegen dortige Rechtsvorschriften verbracht worden ist. Lässt sich nicht klären, ob das Kulturgut nach dem 26.04.2007 verbracht worden ist, so wird widerleglich vermutet, dass das Kulturgut nach diesem Tag aus dem Hoheitsgebiet des Vertragsstaates verbracht worden ist. Bei dem Stichtag handelt es sich um den Zeitpunkt des Inkrafttretens des deutschen Zustimmungsgesetzes zum UNESCO-Übereinkommen vom 20.04.2007 (BGBI. II, S. 626; vgl. Elmenhorst/Wiese, KGSG 2018, § 52 Rn. 14).

Diese Vermutungsregelung kann bereits dem Wortlaut nach nicht erweiternd angewandt werden. Nach diesem greift sie erst dann, wenn die Voraussetzung erfüllt ist, dass das Kulturgut unter Verstoß gegen Rechtsvorschriften des Herkunftsstaates aus diesem verbracht worden ist. Die Beantwortung dieser Frage wird in einer Vielzahl von Fällen - wie auch bei dem vorliegenden - davon abhängig sein, ob das Kulturgut tatsächlich aus dem Herkunftsstaat stammt, ob es die für ein Ausfuhrverbot ausschlaggebenden Merkmale (wie z.B. das Alter) erfüllt und ob die zum Verbringungszeitpunkt geltenden Rechtsvorschriften des Herkunftsstaates überhaupt schon ein Ausfuhrverbot vorsahen.

Die Vermutungsregelung betrifft des Weiteren - ausgehend von ihrer systematischen Einordnung - einen vollkommen anderen Sachverhalt und ist auch aus diesem Grund und schon gar nicht erweiternd - auf die für die Anordnung einer Sicherstellung erforderlichen Voraussetzungen anwendbar. Während die Sicherstellung im Kapitel 4 des Kulturgutschutzgesetzes geregelt ist, welches das Instrumentarium staatlicher Eingriffsmöglichkeiten zur Unterbindung unrechtmäßigen Kulturgutverkehrs reglementiert, befasst sich das Kapitel 5 mit den Rückgabeansprüchen der Herkunftsstaaten.

Schließlich vermag der Beklagte eine Beweislastumkehr auch nicht daraus herzuleiten, dass der das Kulturgut Einführende "sachnäher" sei. Dies zeigt bereits die Erwägung, dass dies - wie der vorliegende Fall auch zeigt - etwa im Hinblick auf den Informationszugang zu den zugrunde zulegenden Rechtsvorschriften des Herkunftsstaats, gerade nicht zutrifft. Ebenso wenig besteht eine rechtliche Handhabe dafür, den Betreffenden auf von Dritten herausgegebenes Informationsmaterial - wie vorliegend die sog. „rote Liste" - zu verweisen.

III. Da der angefochtene Bescheid, mit dem die Sicherstellung der Buddha-Statue angeordnet worden ist, aufzuheben war, war auf Antrag des Klägers der Beklagte zu deren Herausgabe zu verpflichten.

Mit der Aufhebung eines Verwaltungsakts entsteht gem. $\S 113$ Abs. 1 Satz 2 und 3 VwGO kraft materiellen Rechts ein Anspruch des Klägers gegen die Behörde auch auf Beseitigung der unmittelbaren, noch andauernden Folgen von dessen Vollziehung (Kopp/Schenke, VwGO, 25. Aufl. 2019, § 113 Rn. 80 f.), wie z. B. - wie vorliegend - auf Rückgabe der sicher- 
gestellten Sache. Die Voraussetzung hierfür, nämlich, dass die Behörde hierzu in der Lage und Spruchreife gegeben ist (vgl. $\S 113$ Abs. 1 Satz 3 VwGO), ist erfüllt. In diesem Fall ist das Gericht zur Entscheidung verpflichtet. Soweit der Gesetzeswortlaut davon spricht, dass das Gericht die Rückgängigmachung von Vollzugsfolgen anordnen „kann“, bedeutet dies nur, dass das Gericht hierzu - bei Vorliegen der Voraussetzungen - befugt ist, nicht aber, dass die Anordnung in dessen Ermessen steht (Kopp/Schenke, VwGO, 25. Aufl. 2019, § 113 Rn. 93).

IV. Die Kostenentscheidung folgt aus $\S 154$ Abs. 1 VwGO. Das Gericht sieht davon ab, das Urteil hinsichtlich der Kosten für vorläufig vollstreckbar zu erklären (§ 167 Abs. 2 VwGO). Die Voraussetzungen für eine Zulassung der Berufung durch die Kammer sind nicht erfüllt. 